\title{
Is sedimentation container-shape dependent?
}

\author{
C.W. J. Beenakker \\ Department of Chemistry, Stanford University, Stanford, California 94305 \\ P. Mazur \\ Instituut-Lorentz, Rijksuniversitelt te Leiden, Nieuwsteeg 18, 2311 SB Leiden, The Netherlands
}

(Received 29 April 1985; accepted 24 July 1985)

The question is addressed as to the dependence of sedimentation of a homogeneous suspension on the shape of the container. It is demonstrated, by comparing calculations for spherical and plane geometries, that shape-dependent contributions to the sedimentation velocity remain in the limit of infinitely distant container walls. Upon transformation from the laboratory reference frame to a local frame of reference that moves with the average volume velocity, this shape dependence is found to disappear.

\section{INTRODUCTION}

The question of a possible dependence of the sedimentation velocity of suspended particles on the shape of the vessel containing the suspension is of considerable importance, given the wide-spread use of sedimentation measurements for the characterization of suspensions. It is surprising, therefore, that this question has not received much attention in the literature. An important exception is formed by Burgers, who in an extensive study ${ }^{1}$ noted the possibility of the sedimentation velocity being dependent on the shape of the vessel. No definite answer, however, could be obtained.

It should be emphasized that Burgers was speaking of suspensions that are homogeneous within the container, in order to avoid the occurrence of convective flows caused by (horizontal) density gradients. From a macroscopic point of view, these latter flows can simply be understood as being caused by buoyancy ${ }^{2}$ : volume elements of the suspension with the lowest density rise relative to volume elements with the highest density. Obviously, buoyancy-driven convection is absent if the suspension is homogeneous.

How can one understand that the average velocity with which particles in the bulk of the suspension sediment might depend on the shape of the container, no matter how large the container? The possibility of such an effect arises from the long range of so-called hydrodynamic interactions. That is to say, a perturbation of the fluid flow caused by the motion of a particle decays very slowly, in fact only with the inverse first power of the separation to the particle. For this reason, container walls have an essential influence on sedimentation-even if the container is very large. A dramatic illustration of this fact is formed by the divergency of the sedimentation velocity encountered in an unbounded suspension-a paradoxical situation first noticed by Smoluchowski. ${ }^{3}$

In a previous publication ${ }^{4}$ (hereafter referred to as I) the influence of a plane wall supporting the suspension was investigated. Using recently derived formulas ${ }^{5}$ for the mobility tensors of spherical particles in a fluid bounded in one direction by a plane, it was shown that a finite answer for the sedimentation velocity results (in contrast to what is found in an unbounded system), even in the limit of an infinitely distant wall.

In the present paper a similar calculation is performed for a suspension bounded in all directions by a spherical wall, exploiting the circumstance that formulas for the mobilities in such a system are now available. ${ }^{6}$ The results are then compared with those for the plane geometry of Paper I, in order to investigate the issue of the shape dependence of the sedimentation velocity. Finally, the relationship with the work of Batchelor ${ }^{7}$ on sedimentation is discussed.

\section{SEDIMENTATION INSIDE A SPHERICAL CONTAINER A. Results from the hydrodynamic analysis}

We consider the motion of $N$ spherical particles with radii $a_{t}(i=1,2, \ldots, N)$ in an incompressible fluid with viscosity $\eta$, which is bounded by a (motionless) spherical container wall with radius $b$. The centers of the particles have positions $\mathbf{R}_{i}(i=1,2, \ldots, N)$ and lie inside the container. We take the center of the container as the origin of our coordinate system. The motion of the fluid is described by the linear quasistatic Stokes equation, supplemented by stick boundary conditions on the surfaces of the particles and on the container wall.

The velocity $\mathbf{U}_{t}$ of particle $i$ can then be expressed as a linear combination of the forces $\mathbf{K}_{J}$ exerted by the fluid on each particle $j$,

$$
\mathbf{U}_{\imath}=-\sum_{j=1}^{N} \mu_{l j} \cdot \mathbf{K}_{\jmath}, \quad i=1,2, \ldots, N .
$$

(We consider the case of free rotation, i.e., the fluid exerts no torque on each of the particles.) General expressions for the mobility tensors $\boldsymbol{\mu}_{i j}$ for this system have been obtained in Ref. 6 as an expansion in the three parameters $a / b, a / R_{p p^{\prime}}$, and $R_{p} / b$, where $a$ is the typical radius of a particle, and $R_{p p^{\prime}}$ and $R_{p}$ denote, respectively, the typical distances between two particles and between a particle and the center of the container. For the special case of particle $i$ concentric with the container (which is the case we shall consider in the following) one finds

$$
\begin{aligned}
\left.6 \pi \eta a_{i} \boldsymbol{\mu}_{i j}\right|_{\mathbf{R}_{i}=0}= & \mid \delta_{i j}+\left[{ }_{4}^{3} a_{\imath} R_{J}^{-1}\left(1+\hat{r}_{J} \hat{r}_{J}\right)\right. \\
& \left.-\frac{3}{4} a_{\imath}\left(a_{t}^{2}+a_{J}^{2}\right) R_{J}^{-3}\left(\hat{r}_{J} \hat{r}_{J}-\frac{1}{3}\right)\right]\left(1-\delta_{l}\right) \\
& +1\left[-\frac{9}{4} a_{\imath} b^{-1}+\frac{5}{4} a_{\imath}\left(a_{t}^{2}+a_{J}^{2}\right) b^{-3}\right] \\
& +\frac{3}{2} R_{J}^{2} b^{-2}\left(1-\frac{1}{2} \hat{r}_{J} \hat{r}_{J}\right)\left(a_{\imath} b^{-1}-a_{\imath}^{3} b^{-3}\right) \\
& + \text { h.o.t. }
\end{aligned}
$$


Here "h.o.t." stands for "higher-order terms," defined as terms of order $(a / b)^{n}\left(a / R_{p p^{\prime}}\right)^{m}\left(R_{p} / b\right)^{\prime}$ in the expansion parameters, with $n+m \geqslant 4$ and $l$ arbitrary. ${ }^{8}$ We have used the notations $R_{j} \equiv\left|\mathbb{R}_{j}\right|, \hat{r}_{j} \equiv \mathbb{R}_{j} / R_{j} ; \delta_{l j}$ is the $\mathbb{K}$ ronecker delta and $I$ denotes the second rank unit tensor.

The flow of fluid caused by the motion of the particles is described by the velocity field $v(\mathbf{r})$, given by

$$
\mathbf{v}(\mathbf{r})=-\sum_{j=1}^{N} \mathrm{~S}_{j}(\mathbf{r}) \cdot \mathbf{K}_{j} .
$$

The tensor field $S_{j}(\mathbf{r})$ is closely related to the mobilities of the particles, and may in fact be derived from these by considering a "test particle" at point $\mathbf{r}$ of infinitesimal radius [cf. Ref. 6, Eq. (4.9)]. To the same order as the result (2), one thus finds for the value of the field $S$ at the center of the container

$$
\begin{aligned}
6 \pi \eta a_{j} \mathrm{~S}_{J}(\mathbf{r}=0)= & \frac{3}{4} a_{J} R_{J}^{-1}\left(1+\hat{r}_{J} \hat{r}_{J}\right) \\
& -\frac{3}{4} a_{J}^{3} R_{J}^{-3}\left(\hat{r}_{J} \hat{r}_{J}-\frac{1}{3}\right)+\left(1-\frac{9}{4} a_{J} b^{-1}\right. \\
& \left.+\frac{5}{4} a_{J}^{3} b^{-3}\right)+\frac{3}{2} R_{J}^{2} b^{-2}\left(1-\frac{1}{2} \hat{r}_{J} \hat{r}_{J}\right) a_{J} b^{-1} \\
& + \text { h.o.t. }
\end{aligned}
$$

\section{B. Calculation of the sedimentation velocity}

We assume inside the container a homogeneous distribution of identical sedimenting particles ( $a=$ radius, $n=$ number density of the particles), and ask for (i) the average velocity $\mathbf{v}_{p}$ of particles, and (ii) the average velocity $\mathbf{v}_{f}$ of fluid, both evaluated at the center of the container in the limit that its radius goes to infinity. In view of Eqs. (1) and (3) we can write these two quantities as conditional averages of $\boldsymbol{\mu}$ and $S$, respectively, ${ }^{9}$

$$
\begin{aligned}
& \mathbf{v}_{p}=\lim _{b \rightarrow \infty}\left\langle\sum_{J} \boldsymbol{\mu}_{i j} \mid \mathbf{R}_{t}=0\right\rangle \cdot \mathbf{F} \\
& \left.\mathbf{v}_{f}=\lim _{b \rightarrow \infty}\left\langle\sum_{l} \mathrm{~S}_{i}(\mathbf{r}=0)\right| R_{J}>a \text { for all } j\right\rangle \cdot \mathbf{F} .
\end{aligned}
$$

Here $\left\langle\ldots \mid \mathbf{R}_{t}=0\right\rangle$ denotes an average over those configurations of the particles inside the container for which $\mathbf{R}_{i}=0$. Similarly, $\langle\cdots| R_{j}>a$ for all $\left.j\right\rangle$ denotes an average over those configurations for which no particle overlaps the origin (in other words, there is fluid at $\mathbf{r}=0$ ). The gravitational force (corrected for buoyancy) on each of the particles is denoted by $\mathbf{F}=\frac{4}{3} \pi a^{3}\left(\rho_{p}-\rho_{f}\right) \mathbf{g}\left(\rho_{p}=\right.$ mass density of a particle, $\rho_{f}$ $=$ mass density of the fluid, and $\mathbf{g}=$ gravitational acceleration).

Using the results for $\boldsymbol{\mu}$ and $S$ given in Eqs. (2) and (4) we will calculate the average particle and fluid velocities $\mathbf{v}_{p}$ and $\mathbf{v}_{f}$ to linear order in the volume fraction $\phi=\frac{4}{3} \pi a^{3} n$ of the suspended particles.

Substituting Eq. (2) into Eq. (5), one finds for the average particle velocity

$$
\begin{aligned}
\mathbf{v}_{p}= & \lim _{b \rightarrow \infty}\left[1+\mathscr{O}\left(b^{-1}\right)+\phi \int_{2 a}^{b-a}\left(3 a R^{-1}-\frac{27}{4} a b^{-1}\right.\right. \\
& +\frac{15}{2} a^{3} b^{-3}+\frac{15}{4} R^{2} b^{-2}\left(a b^{-1}-a^{3} b^{-3}\right) \\
& \left.+\Delta(R, b)) a^{-3} R^{2} d R+\mathscr{O}\left(\phi^{2}\right)\right](6 \pi \eta a)^{-1} \mathbf{F}
\end{aligned}
$$

where an angular integration has already been carried out. The function $\Delta(R, b)$ appearing in the integrand consists of terms denoted by h.o.t. in Eq. (2). These terms are of order $b^{-n} R-{ }^{-m}(R / b)^{l}$ with $n+m \geqslant 4$ ( $l$ arbitrary). Performing the radial integration in $\mathrm{Eq}$. (7) and subsequently taking the limit $b \rightarrow \infty$ of the result, one finds

$$
\mathbf{v}_{p}=\left[1+\left(-2+\Delta_{\infty}\right) \phi+\mathscr{O}\left(\phi^{2}\right)\right](6 \pi \eta a)^{-1} \mathbb{F}
$$

with the definition

$$
\begin{aligned}
\Delta_{\infty} & \equiv \lim _{b \rightarrow \infty} \int_{2 a}^{b-a} \Delta(R, b) a^{-3} R^{2} d R \\
& =\int_{2 a}^{\infty} \lim _{b \rightarrow \infty} \Delta(R, b) a^{-3} R^{2} d R .
\end{aligned}
$$

The exchange of limit and integration performed here is justified by the fact that $\Delta(R, b)$ decreases sufficiently fast with increasing $R$ and $b$ for the first integral in Eq. (9) to converge uniformly in $b$. [As an aside, we note that the full integral in Eq. (7) does not converge uniformly in $b$. In fact, if one would perform a similar exchange of limit and integration there, one would find a divergent result. This is essentially the Smoluchowski paradox mentioned in the Introduction.] One sees from Eq. (9) that $\Delta_{\infty} \phi$ is the contribution of order $\phi$ to the average particle velocity resulting from short-ranged (i.e., of order $R^{-n}$ with $n \geqslant 4$ ) hydrodynamic interactions of two particles in an unbounded fluid. From the calculations of Batchelor, ${ }^{7}$ we take the result

$$
\Delta_{\infty} \approx-1.55 \text {, }
$$

giving the final answer

$$
\mathbf{v}_{p}=\left[1-3.55 \phi+O\left(\phi^{2}\right)\right](6 \pi \eta a)^{-1} \mathbf{F} .
$$

The calculation of the average fluid velocity at the center of the container proceeds along the same lines. Substitution of Eq. (4) into Eq. (6) gives

$$
\begin{aligned}
\mathbf{v}_{f}= & \lim _{b \rightarrow \infty} \phi \int_{a}^{b-a} a^{-3} R^{2} d R\left(3 a R^{-1}-\frac{27}{4} a b^{-1}\right. \\
& \left.+\frac{15}{4} a^{3} b^{-3}+\frac{15}{4} a R^{2} b^{-3}+\Delta^{\prime}(R, b)\right)(6 \pi \eta a)^{-1} \mathbf{F} .
\end{aligned}
$$

The function $\Delta^{\prime}(R, b)$ used here plays the same role as $\Delta(R, b)$ in Eq. (7), that is to say, $\Delta^{\prime}(R, b)$ consists of terms of order $b^{-n} R^{-m}(R / b)^{l}$ with $n+m \geqslant 4$. As in Eq. (9), we may therefore exchange the limit $b \rightarrow \infty$ with the integration over $R$ to determine the contribution from $\Delta^{\prime}$ to the average fluid velocity. This contribution is then found to vanish in view of the fact that ${ }^{10} \Delta^{\prime}(R, b) \rightarrow 0$ as $b \rightarrow \infty$. A straightforward integration of the remaining terms in Eq. (12) gives, in the limit $b \rightarrow \infty$,

$$
\mathbf{v}_{f}=\left[2 \phi+\mathscr{O}\left(\phi^{2}\right)\right](6 \pi \eta a)^{-1} \mathbf{F} .
$$

Having calculated both the average particle and fluid velocities at the center of the container, we can now determine the average volume velocity $v_{v}$, which is given by

$$
\mathbf{v}_{v}=\phi \mathbf{v}_{p}+(1-\phi) \mathbf{v}_{f} .
$$

The results (11) and (13) give with Eq. (14)

$$
\mathbf{v}_{v}=\left[3 \phi+\mathscr{O}\left(\phi^{2}\right)\right](6 \pi \eta a)^{-1} \mathbf{F} .
$$

Contrary to what one might expect, we thus find that there 
exists a nonvanishing convective flow, although the suspension is homogeneous, so that no buoyancy-driven convection can occur. We note that, because of incompressibility of the suspension, the volume flux through any closed surface must vanish. Our result (15), that there is a nonzero volume velocity at the center of the container, therefore implies the existence of a vortex of convective flow inside the spherical container.

We conclude this section by briefly considering the case of a vertically inhomogeneous suspension: we assume that the number density of the particles inside the container is constant (equal to $n$ ) for $z<f b$, and equal to zero for $z>f b-$ where we have introduced the filling parameter $f(0<f<1)$. (The coordinate system is such that the center of the container is in the plane $z=0$ and the gravitational acceleration is in the minus- $z$ direction.) Since the calculations are very similar to those described above for the case of a completely homogeneous suspension, we only give the results

$$
\begin{aligned}
\mathbf{v}_{p}= & {\left[1+\left(-\frac{7}{2}-1.55+\frac{9}{4} f-\frac{3}{4} f^{3}\right) \phi+\mathscr{O}\left(\phi^{2}\right)\right] } \\
& \times(6 \pi \eta a)^{-1} \mathbf{F}, \\
\mathbf{v}_{f}= & {\left[\left(\frac{1}{2}+\frac{9}{4} f-\frac{3}{4} f^{3}\right) \phi+\mathscr{O}\left(\phi^{2}\right)\right](6 \pi \eta a)^{-1} \mathbf{F}, } \\
\mathbf{v}_{v}= & {\left[\left(\frac{3}{2}+\frac{9}{4} f-\frac{3}{4} f^{3}\right) \phi+\mathscr{O}\left(\phi^{2}\right)\right](6 \pi \eta a)^{-1} \mathbf{F} . }
\end{aligned}
$$

In the limit $f \rightarrow 1$, the previous Eqs. (11), (13), and, (15) are recovered. It is noteworthy that, although the average particle velocity $\mathbf{v}_{p}$ at the center of the container depends on the filling parameter $f$, the quantity $\mathbf{v}_{p}-\mathbf{v}_{v}$ is independent of $f$.

\section{CONCLUSIONS AND DISCUSSION}

The main resuits from the calculations of Sec. II are collected in Table I, together with the results from Paper I. By comparing the values of $\mathbf{v}_{p}$ in the two container geometries, we can conclude that the average velocity of a sedimenting particle in a homogeneous suspension depends on the shape of the container, even in the limit of infinitely distant container walls. However, if one transforms from the laboratory reference frame (this is the reference frame in which the container is at rest) to a local frame of reference that moves with the average volume velocity $\mathbf{v}_{v}$, this shape dependence disappears. That is to say, the quantity $\mathbf{v}_{p}-\mathbf{v}_{u}$ is the same for the two container geometries studied, although $\mathbf{v}_{p}$ is not.

Batchelor ${ }^{7}$ argued that the velocity of sedimentation relative to the average volume velocity should be independent

TABLE I. Average particle velocities $\mathbf{v}_{p}$, fluid velocities $\mathbf{v}_{f}$, and volume velocities $\mathbf{v}_{u}$ for (i) a homogeneous sedimenting suspension bounded in the direction of gravity by a plane wall, ${ }^{4}$ and (ii) a homogeneous suspension enclosed by a spherical wall (with velocities evaluated at the center of the container). In both cases the limit is taken of infinitely distant container walls. [We have defined $\mathbf{v}_{0}=(6 \pi \eta a)^{-1} \mathbf{F}$.]

\begin{tabular}{llcccc}
\hline \hline & $\begin{array}{l}\text { Container } \\
\text { geometry }\end{array}$ & $\mathbf{v}_{p}$ & $\mathbf{v}_{f}$ & $\mathbf{v}_{v}$ & $\mathbf{v}_{p}-\mathbf{v}_{v}$ \\
\hline (i) & Plane & $(1-6.55 \phi) \mathbf{v}_{0}$ & $-\phi \mathbf{v}_{0}$ & 0 & $(1-6.55 \phi) \mathbf{v}_{0}$ \\
(ii) Sphere & $(1-3.55 \phi) \mathbf{v}_{0}$ & $2 \phi \mathbf{v}_{0}$ & $3 \phi \mathbf{v}_{0}$ & $(1-6.55 \phi) \mathbf{v}_{0}$ \\
\hline
\end{tabular}

of the shape of the container, on the basis of general considerations of a physical nature, which are valid for the unbounded system. Ultimately, these considerations should follow from an evaluation of the influence of container walls on the mobilities of the sedimenting particles. Such an explicit calculation, confirming Batchelor's result, was performed in this paper for a spherical container geometry.

That the sedimentation velocity in the laboratory reference frame is not container-shape independent is a new result, which goes beyond Batchelor's theory. ${ }^{7}$

The occurrence of convective flows in homogeneous suspensions, demonstrated in this paper for the case of a spherical container, is a surprising phenomenon, of a different nature than the well-known buoyancy-driven convection that occurs in inhomogeneous suspensions. ${ }^{2}$ We will refer to the former convective flow as essential convection. We emphasize here the different order of magnitude of the two convective phenomena: at very small volume fractions, say $\phi \sim 10^{-4}$, essential convection is negligibly small $\left(\mathbf{v}_{v} \sim 10^{-4} \mathbf{v}_{0}\right)$, whereas buoyancy-driven convective flows of order $10 v_{0}$ have been observed ${ }^{2}$ at these volume fractions.

For this reason the spherical container geometry, considered in this paper because of its theoretical simplicity, is not well suited for the experimental observation of essential convection. Its occurrence would be masked completely by buoyancy-driven convective flows that would arise when, after a short time, the inclined boundary walls have induced horizontal density gradients. ${ }^{2}$ However, we surmise that essential convection is not limited to containers with inclined walls (such as the spherical container studied in this paper), but is rather a general phenomenon in systems without translational invariance in a horizontal plane.

Consider what would happen if such a convective flow arises in a homogeneous suspension sedimenting in a vertical cylinder; the flow itself then causes horizontal density gradients to develop as particles sediment faster near the centerline of the cylinder rather than further outward. (In particular, an initially flat horizontal interface separating clear fluid from the suspension will with time become indented at its center.) Since we expect the inhomogeneities to be localized near the top and bottom of the suspension, it seems rather unlikely that the flow in the middle part of a long cylinder would be very much affected by the resulting buoyancy-driven convection (which, at the top of the suspension, tends to flatten the interface). In fact, a weak convective vortex in a vertical cylinder (with a downward velocity on the centerline) has been reported by Kinosita, ${ }^{11}$ and cannot be accounted for by buoyancy-driven convection alone. Essential convection of the type calculated in this paper could well be the explanation of that observation.

\section{ACKNOWLEDGMENTS}

We thank the referee for correcting an oversight in the manuscript.

Carlo Beenakker gratefully acknowledges a fellowship from the Niels Stensen Stichting.

'J. M. Burgers, Proc. K. Ned. Akad. Wet. 44, 1045, 1177 (1941); 45, 9, 126 $(1942)$. 
${ }^{2}$ See, for example, W D Hill, R R Rothfus, and K Li, Int J Multiph Flow 3, 561 (1977), and references therein

${ }^{3} \mathrm{M}$ Smoluchowski, Proceedings of the 5th International Congress of Mathemattcians, edited by E W Hobson and A E H Love (Cambridge U P Cambridge, 1913), Vol 2, p 192

${ }^{4} \mathrm{C}$ W J Beenakker and P Mazur, Phys Fluıds 28, 767 (1985) (Paper I) ${ }^{5} \mathrm{C}$ W J Beenakker, W van Saarloos, and P Mazur, Physica 127A, 451 (1984)

${ }^{6} \mathrm{C}$ W J Beenakker and P Mazur, Physica 131A, 311 (1985)

${ }^{7} \mathrm{G}$ K Batchelor, J Fluid Mech 52, 245 (1972)

${ }^{8}$ Equation (2) results from Eq (5 1) of Ref 6, with the addition of the term denoted by ${ }^{3} \mathrm{~A}_{r 0}^{(13)} \odot \mathrm{B}^{\left(3{ }^{31}\right.} \odot^{1} \mathrm{~A}_{0,}^{(3)}{ }^{1)}$ in that paper The order of the terms not exphcitly written down in $\mathrm{Eq}$ (2) follows from the general expression for the mobility given in Ref 6 [Eq (4 2)], making essential use of the fact that $\mathbf{R}_{t}=0$

${ }^{9}$ As in Paper I we tgnore the influence of Brownian motion on sedimentation, of note 13 in I

${ }^{10}$ This limit expresses the well-known fact that the fluid velocity field caused by the motion of one spherical particle in an unbounded fluid does not contain terms of order $R^{-n}$ with $n \geqslant 4$ (Here $R$ is the distance to the particle)

${ }^{11} \mathrm{~K}$ Kinosita, J Collord Sci 4, 525 (1949) 